\title{
Gradual Weight Loss Does not Prevent Hypohydration during Preparation Period in Elite Kickboxer of Croatia
}

\author{
Damir Zubac, Luka Tomic and Hrvoje Karnincic \\ Department of Combat Sports, Faculty of Kinesiology, University of Split, Split 21000, Croatia
}

\begin{abstract}
According to present combat sports literature, acute dehydration is the most common weight-manipulating technique adopted by combat sport athletes. Over a one-month period, possible fluctuations in dehydration assessment biomarkers of elite kickboxing athletes $(N=11)$ are investigated. Using repeated measures $(\mathrm{RM})$ ANOVA, we found significant differences in body mass (BM) $(1.2 \% \Delta)$ irrespective of stability in urine specific gravity (USG) and total body water values (TBW\%) between trials. RM ANOVA revealed significant differences among the three trials in BM\% $\Delta(18.73 ; \mathrm{df}=2 P<0.000)$, whereas changes in body fat (BF\%) revealed borderline values $(5.61, \mathrm{df}=2 P<0.02)$. Post-hoc analyses demonstrated significant differences between $\mathrm{t}-1$ and $\mathrm{t}-2(P<$ $0.001)$, and $\mathrm{t}-1$ and $\mathrm{t}-3(P<0.001)$, in BM\% $\Delta$. Correlation analyses between hydration biomarkers and respective BM\% are weak and trivial, indicating non-significant association $(\mathrm{r}=0.03-0.26, P$-values $>0.05)$. Hypohydration is undoubtedly overlooked by athletes and coaches in kickboxing. Notably, current weight class categories (WCC) stratification may be a pathway that facilitates the temptation of kickboxing athletes to migrate into lower WCC. Endorsement of novel and effective control mechanism besides morning weight is warranted. However, this topic remains a controversial issue and deserves special attention.
\end{abstract}

Key words: Dehydration, martial arts, preparation period, urine index.

\section{Introduction}

In recent years, there has been a growing debate in terms of the need to develop policies aimed to monitor aggressive weight reduction in combat sports. According to current scope of combat sports research, body mass $(\mathrm{BM})$ reduction prior to competition is achieved exclusively by voluntary acute dehydration [1]. Voluntary dehydration, the most frequent weight-reducing method adopted by combat athletes, occurs as a technique to gain a real or perceived competitive advantage [2]. However, detrimental negative health consequences, including death, are documented as a result of voluntary dehydration. Nevertheless, this practice is still common in the majority of combat sports [3].

To prevent recurrence of tragic death related to vigorous weight reducing attempts, the National Collegiate Athletic Association (NCAA) governing authorities introduced new weight-certification rules in

Corresponding Author: Hrvoje Karnincic Ph.D., professor, research fields: combat sports, physiology and sport history.
1998 to discourage health-threatening behavior adopted by colleague wrestlers [4]. Application of new rules also incorporated the determination of hydration status on competition day addressed by urine specific gravity (USG) assessment. However, other combat sports, such as kickboxing still fail to recognize any kind of effective control mechanism beside official weigh-in, despite numerous articles indicating the violation of USG cut-off assumptions for athletes (i.e., > $1.020 \mathrm{~g} / \mathrm{ml}^{-1}$ ) in combat sports on competition day. More precisely, some authors [5-7] recently reported high prevalence of serious hypohydration solely on competition day via USG assessment.

Meanwhile, majority of the investigations failed to collect data during preparation period; therefore, a coherent picture of hydration status is lacking. Impaired physiological processes and health risks associated with harmful weight reduction protocols [3] justify the need for screening and detecting subsequent dehydration in combat sports athletes, not exclusively on competition day, but during preparation period as 
well. It is of practical importance for combat athletes to reduce adverse and health-threatening side effects induced by different approaches to achieve competition weight. Our perspective research on hydration assessment applied to combat sports shows a lack in multidimensional approach to the issue.

Therefore, the purpose of the present study was twofold. Firstly, to examine changes in dehydration assessment biomarkers during preparation for major competition in real-life settings. Secondly, we also examined association of hydration assessment biomarkers to possible changes in body mass (BM) during preparation period. We hypothesized that change in dehydration assessment biomarkers would occur and significantly reflect upon potential BM fluctuations prior to competition.

\section{Experimental procedures}

This study was approved by the authors' Institutional Research Ethical Board in accordance with the Helsinki Convention, prior to any data collection.

\subsection{Participants}

The participants included in this study were elite youth and senior kickboxing athletes, current national champions of Croatia ( $n=11 ; 10$ males, 1 female athlete, $22.1 \pm 4.1$ years of age $185.6 \pm 9.8 \mathrm{~cm}$ height, $83.4 \pm 15.8 \mathrm{~kg}$ body mass, $24.5 \pm 3.2 \mathrm{BMI}, 5.9 \pm 2.8$ years of training experience, $12.5 \pm 2.6$ hours of training per week). Participation was voluntary for all athletes. National team manager and athletes provided written informed consent prior to any data collection. Participants had no previous history of renal or urinary tract disease, according to the national team medical staff. The selection criteria were: (1) must be a current national champion with international competition experience (> 25 fights); (2) negative anti-doping results. Not only because of the specificity of physiological adaptations of elite combat athletes to weight reduction [1], but also to obtain ecological validity of research, strict criteria in the selection of participants is established. The participants and coaches were informed in detail about the experimental procedures and the possible risks and benefits of the project.

\subsection{Experimental Approach to the Problem}

Our study was carried out during a one-month of training camp in Split, Croatia, prior to a major international competition. During study enrollment, before any data collection, some instructions were given to athletes and their coaches regarding weight management practice. Athletes did not train for 24 hours before the first weigh-in. The national team manager agreed that during the first week of preparation all participants would follow the same training, diet and rest pattern. The participants were asked to withdraw from all dietary sources of caffeine, and instructed to maintain their normal diet and training, without any weight reduction practices or thermal strain exposure 1 week prior to the first data collection. Our study design did not interfere with costume training regime, or weight reduction practice after the first hydration assessment. The temperature and relative humidity in the testing facility ranged from $15^{\circ} \mathrm{C}-18^{\circ} \mathrm{C}$ and $45 \%-55 \%$, for all trails, respectively.

\subsection{Experimental Trial}

Athletes were tested at three different times: during an ordinary training period (1) when the athletes were at their normal BM (t-1); (2) 10 days before (t-2), and (3) 3 days before an official competition (t-3). Evening before the urine sampling, all participants were provided with one sterile container to collect a morning first urine specimen. In agreement with American College of Sports Medicine's (ACSM) hydration testing guidelines [8], each participant was instructed to provide a small urine sample collected mid-flow from the first void in the morning on the assessment day of each trial. USG has been suggested as a superior index to detect hypohydration following overnight 
sleep without fluid intake [9]. The cut-off of urinary indexes of hydration status was based on ACSM guidelines [8]: USG $<1.010 \mathrm{~g} / \mathrm{ml}^{-1}$ indicates a well-hydrated state, USG $=1.010-1.020 \mathrm{~g} / \mathrm{ml}^{-1}$ indicates minimal hypohydration, USG $>1.021 \mathrm{~g} / \mathrm{ml}^{-1}$ indicates significant hypohydration, and USG $>1.030$ $\mathrm{g} / \mathrm{ml}^{-1}$. We selected a morning first USG $>1.020 \mathrm{~g} / \mathrm{ml}^{-1}$ values as a to be point toward a state of hypohydration, based on the suggestion of Sawka et al. [8].

\subsubsection{Anthropometric Measurement}

The percentage of $\mathrm{BM}$ expressed as fat $(\% \mathrm{BF})$ and was estimated according to the 3 -site skinfold method (triceps, subscapular, and abdominal) using a Harpenden caliper, whereas body height was measured by a Martin's anthropometer [10].

2.3.2 Bioelectrical Impedance Analysis Assessment

Bioelectrical impedance analysis (BIA) presents a highly portable, low cost noninvasive alternative technique, commonly applied to combat sports research that can be used to estimate total body water. The content of body fluids in the body composition was assessed using a Maltron BF-907 Body Composition Analyzer (Essex, UK) at $50 \mathrm{kHz}$. Previous articles in elite amateur boxing suggest that supine body position single-frequency BIA assessment is a reliable hydration status assessment technique [1]. This noninvasive method has been validated against the accepted criterion standard technique (dilution of deuterium-labeled water); it is less laborious and costs less than the criterion standard [11]. Bioelectrical impedance analyzer was used to estimate total body water (\% TBW) according to the standard tetra-polar, the whole-body technique. After 10 minutes of resting in a supine position with hands and legs slightly apart, skin was cleansed by an alcohol, four surface electrodes were placed on the right hand and foot (two placed on the hand and two on the foot) and the displayed values were recorded. Data was demonstrated in percentage rather than absolute units of measurement [12].

\subsubsection{Urinary Analysis}

Participants handed out their first morning urine specimens before weigh-in, and approached the anthropometric measurement. Urine specimens were immediately analyzed in duplicate USG. The final value for each assessment was the average of the two trials. We used an AtagoPal 10s refractometer (Tokyo, Japan) providing accurate readings to 0.001 units. The refractometer was calibrated with distilled water before usage. A glass pipette was used to apply the urine sample to the instrument and the value was digitally read. Once values were recorded, the urine sample was discarded.

\subsubsection{Body Mass}

Participants BM was determined by using official Tanita BC 520 scale while they were barefoot, wearing only dry underwear, in accordance with recommendations of Carlton et al. [13].

\subsubsection{Statistics}

Statistica v.12.0 (StatSoft, Tulsa, OK, SAD) software was used for all calculations. We analyzed data using descriptive statistics of the distribution of variables (mean and standard deviation), and where appropriate, 95\% confidence intervals. For all parameters, a normal distribution was checked with the Shapiro-Wilk test for small samples. Changes in TBW\% and $\mathrm{BM} \%$ were expressed as a percentage of the baseline value. The sphericity (Greenhouse-Geisser adjustment) assumption of the ANOVA was checked with Mauchly's test. By using Bonfferonni correction, the level of significance $\alpha=0.05$ was divided by $\alpha / 3$ yielding a type I error rate of 0.0167 for post hoc test. The statistical significance of the difference between trails for all dependent variables (USG, \% TBW, BM \% $\Delta, \% \mathrm{BF}$ ) was determined using one-way repeated measure (RM) ANOVA. For not-normally distributed data (USG values in the first trial), the nonparametric Friedman test was applied. Bonferroni post hoc was applied if significant difference occurred between trials. Pearson's linear correlation coefficient was calculated between the t-3 values of dehydration assessment biomarkers (USG, \% TBW) and percentage of changes in $\mathrm{BM} \% \Delta$. The effect size (partial-eta squared) was 
calculated in accordance with Hopkins [12]. For revealing the significance of differences, power of analysis was set at the level of $>0.80$. Statistically significant differences were set at $P<0.001$.

\section{Results}

All observed variables passed the Shapiro-Wilk normality test; however, as USG values violated normality assumption ( $\mathrm{t}-1$ ), nonparametric ANOVA test was applied. Reliability results for all variables showed coefficient of variation $(\mathrm{CV})$ values of $0.3 \%$ and $0.5 \%$, and intraclass correlation coefficient (ICCs) of 0.94 and 0.96 , respectively.

One-way RM ANOVA revealed significant differences among the three trials in BM\% $(18.73 ; \mathrm{df}=$ $2 P<0.000 ; \eta^{2-}$ partial eta squared $\left.=0.68\right)$; whereas change in $\mathrm{BF} \%$ by using one-way RM ANOVA revealed borderline values $\left(5.61\right.$, df $2 P<0.02 \eta^{2}-$ 0.40). Post hoc analyses revealed significant differences between $\mathrm{t}-1$ and $\mathrm{t}-2(P<0.001)$, and $\mathrm{t}-1$ and t-3 $(P<0.001)$, in $\mathrm{BM} \% \Delta$. Effect size was moderate and high for all variables (Table 1). Second, by using one-way RM ANOVA to assess dehydration biomarkers, we found no significant difference between USG and TBW\% values between trials irrespective of $\mathrm{BM} \% \Delta$. After a significant $\mathrm{F}$ test, pairwise differences were identified using Bonnferoni's post hoc procedure.

Table 1 Mean and standard deviation (data are presented as mean \pm standard deviation) in studied characteristics of the analyzed athletes.

\begin{tabular}{llllll}
\hline Variable & $\begin{array}{l}\mathrm{t}-1 \\
(n=11)\end{array}$ & $\begin{array}{l}\mathrm{t}-2 \\
(n=11)\end{array}$ & $\begin{array}{l}\mathrm{t}-3 \\
(n=10)\end{array}$ & $\mathrm{ES}\left(\eta^{2}\right)$ & $\beta$ \\
\hline USG* $^{2}$ & $1.024 \pm 0.006$ & $1.023 \pm 0.007$ & $1.026 \pm 0.004$ & -- & -- \\
BIA & $65.5 \pm 2.57$ & $65.33 \pm 2.42$ & $65.89 \pm 1.75$ & -- & -- \\
BM\% $\Delta$ & $83.74 \pm 15.19$ & $83.67 \pm 15.4^{\mathrm{b}}$ & $82.78 .3 \pm 16.3^{\mathrm{c}}$ & 0.68 & 0.96 \\
BF\% & $13.5 \pm 4.1$ & $12.98 \pm 3.99^{\mathrm{b}}$ & $13.01 \pm 3.91^{\mathrm{c}}$ & 0.40 & 0.79 \\
\hline
\end{tabular}

${ }^{\mathrm{a}}$ significant difference between $\mathrm{t}-1$ and $\mathrm{t}-2(P<0.00) ;{ }^{\mathrm{b}}$ significant difference between $\mathrm{t}-2$ and $\mathrm{t}-3(P<0.01) ;{ }^{\mathrm{c}}$ significant difference between t-1 and t-3 $(P<0.01) ;{ }^{¥}$ differences were calculated by means of one-way ANOVA; by using Bonfferonni correction the level of significance $\alpha=0.05$ was divided $\alpha / 3$ yielding a type I error rate of 0.0167 for post hoc ANOVA, ES—effect size, *denotes Fridemann ANOVA — non parametric data distribution.

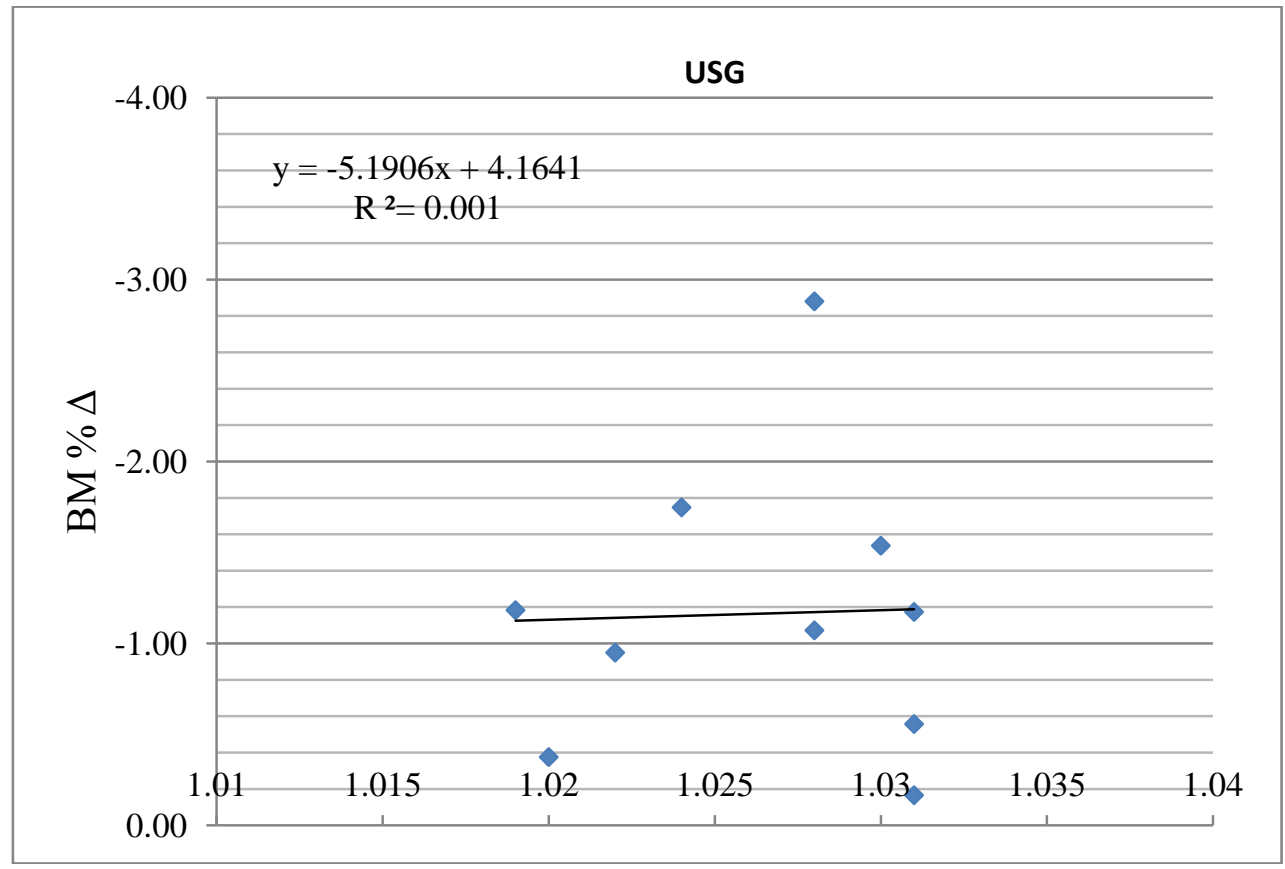

Fig. 1 Association between rate of body mass reduction and urine specific gravity. 


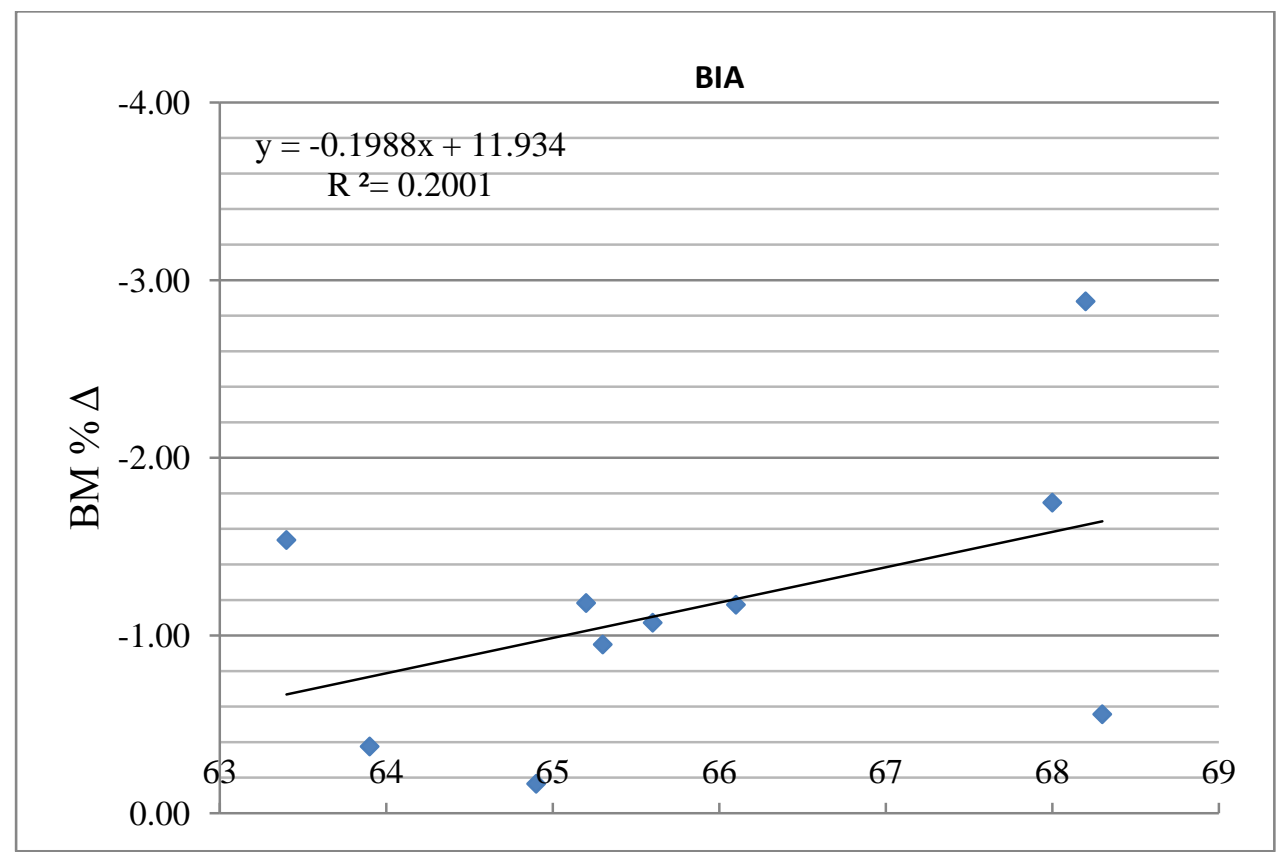

Fig. 2 Association between rate of body mass reduction and BIA.

Correlation analyses between hydration biomarkers at $\mathrm{t}-3$ and respective $\mathrm{BM} \%$ were weak and trivial, revealing non-significant associations $(\mathrm{r}=0.03-0.26$, $P$-values $>0.05$ ) (Figs. 1 and 2). The existence of large effects of the independent variables on both dependent variables (Table 1) suggests that in addition to statistical differences, there is practical significance of the results found for the selected sample of respondents. In accordance with the power of the test $(\beta=0.80)$, high values of $\beta$ were detected (Table 1). The power of the study indicates the possibility of a real chance of discovering the real effects of weight class and the impact of weight class on hydration state. Consequently, the data presented in this study are important, and these differences become noteworthy.

\section{Discussion}

There are several most important findings of this study that are discussed in the following text. Firstly, we found no significant difference between USG and TBW\% values between three trials. On the other hand, we found significant change in BM. Finally, estimation of $\% \mathrm{BF}$ was found slightly, but no significantly decreased.
Urine specific gravity might offer a viable method for field determination of hydration status, and is even suggested as fairly sensitive to detect the fluid deficit according to Hamouti et al. [9]. Basic human physiology mechanisms indicate that subsequent hypohydration would elevate antidiuretic hormone-reducing water excretion in urine and by doing so raise USG values. Casa et al. suggested that a level of dehydration of about $3 \%-5 \%$ body weight would correspond to average urinary index values found in our study (Table 1) and thereby illustrate significant dehydration [14]. Vice versa, we found only $1.2 \%$ of BM change between trials. Evidently, a lack of association $(\mathrm{r}=0.03, P>0.05)$ between USG and $\%$ $\mathrm{BM}$ change was found, especially for those athletes who reduced the most BM (i.e., 4 athletes with USG of 1.030-1.032 $\mathrm{g} / \mathrm{ml}^{-1}$ ). Nevertheless, the main criticism of the use of urinary indexes for dehydration is that urine does not respond as fast or as accurately as blood-to-body fluid deficit based on findings of Popowski et al. [15], therefore, urinary indicators of hydration may lag behind other fluid compartments. Notably, our findings are in line with observations reported in Turkish youth Tae-kwon-do athletes. Kutlu 
and Guler reported a slight prevalence of hypohydration by average values of urine osmolality $998 \pm 171 \mathrm{mOsmol} / \mathrm{kg}^{-1}$ during the preparation period [16].

However, currently suggested cut-off point for USG should not be generalized. Commonly, combat sports research articles show that USG values violate cut-off point assumptions of hydration testing. More precisely, Shirreffs and Maughan reported boxers and wrestlers, when compared to and non-WCC athletes, have higher first morning urine osmolality values (i.e., $775 \pm 263$ vs. $627 \pm 186 \mathrm{mOsmollkg}^{-1} \mathrm{H}_{2} \mathrm{O} ; P<0.05$ ) [17]. However, it is possible that USG may not only reflect body water deficit but be influenced by the clearance of metabolites in urine. Thus, field determination of hydration status could be misleading, and it is still a controversy among combat athletes. Possibly, most subjects function at a minimal level of hypohydration at baseline and accomplished further weight loss rapidly through voluntary dehydration a few days prior to competition as suggested by Smith for elite boxers of England, reducing between $1.7 \%$ to $2.7 \%$ over the final 24 hours [18].

Our data quite clearly illustrate the absence of TBW\% alterations between three trials (Table 1) regardless of differences in $\mathrm{BM}$ values $\left(P<0.001, \eta \mathrm{p}^{2}=0.68\right)$. Baker suggested that $\mathrm{BM}$ change $(\% \Delta)$ is an accurate and reliable index to address the alterations in hydration status [19]. Notably, changes in body weight do not account for athletes who are subsequently dehydrated on their initial preactivity measure [13]. Nevertheless, BIA values highly correlate with total body measures made using isotope dilution under controlled laboratory conditions in euhydrated subjects, although it significantly underestimates the level of absolute fluid losses and is independently altered by changes in body fluid volume and tonicity [11]. This hypothesis logically explains our findings of weak association between BIA and BM \%. However, our findings of BIA (Table 1) are to some extent in line with observations of Reljic et al. [1], but irrespective to our previous findings of single-frequency BIA [7]. Hence, applicability of BIA assessment requires further investigation, whereas assessment of hydration status via BM change in combat athletes who are custom to habitual BM reduction, remains a controversial issue.

It appears that most of our subjects functioned at a minimal level of dehydration at baseline and accomplished further weight loss rapidly through dehydration as found in NCAA wrestlers [20]. However, kickboxing combat athletes are stratified into 12 different WCC by world governing body of full-contact kickboxing [21]. WCC span is relatively narrow when compared to other combat athletes; therefore, kickboxers could be considered susceptible to voluntary weight manipulation protocols. Thus, for kickboxing athletes BM control may be as important an issue as performance [22]. Moreover, Hoffman and Maresh suggested that chronic dehydration might occur in athletes who perform repeated bouts of intense training the same day or on consecutive days [23]. Meanwhile, recent findings show that official weigh-in on competition day did not prevent hypohydration in elite Olympic combat sport athletes [6]. Therefore, current WCC stratification may be a pathway that facilitates the temptation of athletes to migrate into lower WCC. This hypothesis logically explains our findings. Nevertheless, this topic remains a controversial issue and deserves special attention. Notably, it has been recognized that combat athletes habitually consume a diet below current sport nutrition recovery guidelines [6]; therefore, our hypothesis could be considered pertinent to such claims. Sustainably decreased values BF\%, as well as $1.2 \%$ of BM $\Delta$ that reduced over three time trials may implicate gradual weight loss pattern, according to Koral and Dosseville [22].

\section{Conclusion}

The aim of the study was to examine changes in dehydration assessment biomarkers during preparation 
period for major competition in real-life settings and to examine association of hydration assessment biomarkers with possible changes in BM during preparation period. We found no significant difference between USG and TBW\% values between three trials; the significant change was found in $\mathrm{BM}$, while $\% \mathrm{BF}$ was decreased slightly, but no significantly. Only $1.2 \%$ of BM change between trials was found and a lack of association between USG and \% BM change. Thus, serious hypohydration is undoubtedly overlooked by kickboxing athletes. Assessment of hydration status via BM change in combat athletes, who are custom to habitual BM reduction, remains a controversial issue.

\section{Limitations and Strengths}

The restricted number of participants in our study is a limitation. Records were obtained from 10 participants only (female athlete left our study because of injury sustained between $\mathrm{t}-1$ and $\mathrm{t}-2$ ). Moreover, we did not account for pathological changes in urine or dietary intake.

We can vouch for internal validity maintenance during our research. Our participants maintained the same training pattern during our research without any endorsement of prohibited weight reduction methods (i.e., diuretics).

\section{Practical Application}

This investigation demonstrates that prevalence of serious hypohydration is undoubtedly overlooked by kickboxing athletes. WCC span is relatively narrow in kickboxing when compared to other combat athletes; therefore, athletes could be considered susceptible to voluntary weight manipulation. Facing the midpoint of two WCCs and competing in 12 different WCCs might facilitate the consequential temptation to reduce BM to obtain a lower WCC and therefore perhaps become chronically dehydrated, in an attempt to maintain a consistent BM. Therefore, nutrition and hydration strategies should be provided by trained experts in promoting adequate replenishment of nutrients during intense preparation. Endorsement of novel and effective control mechanism to provide immediate feedback to athletes and coaches, beside morning weight, is warranted in combat sports. Notably, in our perspective, a multi-dimensional approach is required when addressing hydration status. In addition, some physiological mechanisms underlying diagnostic accuracy of noninvasive hydration markers phenomenon remain ambiguous. Therefore, careful standardization of all procedures to address hydration via urinary index should be maintained.

\section{References}

[1] Reljic, D., Hassler, E., Jost, J., and Friedmann-Bette, B. 2013. "Rapid Weight Loss and the Body Fluid Balance and Hemoglobin Mass of Elite Amateur Boxers.” Journal of Athletic Training 48 (1): 109-17.

[2] Oppliger, R. A., Magnes, S. A., Popowski, L. A., and Gisolfi, C. V. 2005. "Accuracy of Urine Specific Gravity and Osmolality as Indicators of Hydration Status." International Journal of Sport Nutrition and Exercise Metabolism 15 (3): 236-51.

[3] Franchini, E., Brito, C. J., and Artioli, G. G. 2012. "Weight Loss in Combat Sports. Physiological, Psychological and Performance Effects." Journal of the International Society of Sports Nutrition 9 (1): 52.

[4] Loenneke, J. P., Wilson, J. M., Barnes, J. T., and Pujol, T. J. 2011. "Validity of the Current NCAA Minimum Weight Protocol: A Brief Review." Annals of Nutrition and Metabolism 58 (3): 245-9.

[5] Fernandez-Elias, V. E., Martinez-Abellan, A., Lopez-Gullon, J. M., Moran-Navarro, R., Pallares, J. G., De la Cruz-Sanchez, E., and Mora-Rodriguez, R. 2014. "Validity of Hydration Non-Invasive Indices during the Weightcutting and Official Weigh-in for Olympic Combat Sports.” PLOS One 9 (4): e95336.

[6] Pettersson, S., and Berg, C. M. 2014. "Hydration Status in Elite Wrestlers, Judokas, Boxers, and Taekwondo Athletes on Competition Day." International journal of sport nutrition and exercise metabolism 24 (3): 267-75.

[7] Zubac, D., Karnincic, H., and Zaja, M. 2015. "Hydration Status Assessment among Elite Youth Amateur Boxers." The Journal of Sports Medicine and Physical Fitness. (in press)

[8] Sawka, M. N., Burke, L. M., Eichner, E. R., Maughan, R. J., Montain, S. J., and Stachenfeld, N. S. 2007. "Exercise and Fluid Replacement." Medicine and Science in Sports and Exercise 39 (2): 377-90. 
[9] Hamouti, N., Del Coso, J., and Mora-Rodriguez, R. 2013. "Comparison between Blood and Urinary Fluid Balance Indices during Dehydrating Exercise and the Subsequent Hypohydration When Fluid Is not Restored." European Journal of Applied Physiology 113 (3): 611-20.

[10] Lohman, T. G. 1981. "Skinfolds and Body Density and Their Relation to Body Fatness: A Review." Human Biology 53 (2): 181-225.

[11] Bozzetto, S., Piccoli, A., and Montini, G. 2010. "Bioelectrical Impedance Vector Analysis to Evaluate Relative Hydration Status.” Pediatric Nephrology 25 (2): 329-34.

[12] Hopkins, W. G. 2000. "Measures of Reliability in Sports Medicine and Science." Sports Medicine 30 (1):1-15.

[13] Carlton, A., and Orr, R. M. 2015. "The Effects of Fluid Loss on Physical Performance: A Critical Review." Journal of Sport and Health Science. (in press)

[14] Casa, D. J., Armstrong, L. E., Hillman, S. K., and Montain, S. J. 2000. "National Athletic Trainers' Association Position Statement: Fluid Replacement for Athletes." Journal of Athletic Training 35 (2): 212-24.

[15] Popowski, L. A., Oppliger, R. A., Lambert, G. P., Johnson, R. F., Johnson, A. K., and Gisolfi, C. V. 2001. "Blood and Urinary Measures of Hydration Status during Progressive Acute Dehydration." Medicine and Science in Sports and Exercise 33 (5): 747-53.

[16] Kutlu, M., and Guler, G. 2006. "Assessment of Hydration Status by Urinary Analysis of Elite Junior Taekwon-Do
Athletes in Preparing for Competition." Journal of Sports Sciences 24 (8): 869-73.

[17] Shirreffs, S. M., and Maughan, R. J. 1998. "Urine Osmolality and Conductivity as Indices of Hydration Status in Athletes in the Heat." Medicine and Science in Sports and Exercise 30 (11): 1598-602.

[18] Smith, M. S. 2006. "Physiological Profile of Senior and Junior England International Amateur Boxers." Journal of Sports Science and Medicine 5 (Cssi): 74-89.

[19] Baker, L. B., Lang, J. A., and Kenney, W. L. 2009. "Change in Body Mass Accurately and Reliably Predicts Change in Body Water After Endurance Exercise." European Journal of Applied Physiology 105 (6): 959-67.

[20] Lingor, R. J., and Olson, A. 2010. "Fluid and Diet Patterns Associated with Weight Cycling and Changes in Body Composition Assessed by Continuous Monitoring throughout a College Wrestling Season." Journal of Strength and Conditioning Research 24 (7): 1763-72.

[21] World Association of Kickboxing Organizations. 2015. Competition Rules and Interpretation. Accessed June 28, 2015. http://www.wakoweb.com/en/

[22] Koral, J., and Dosseville, F. 2009. "Combination of Gradual and Rapid Weight Loss: Effects on Physical Performance and Psychological State of Elite Judo Athletes." Journal of Sports Sciences 27 (2): 115-20.

[23] Hoffman, J. R., and Maresh, C. M. 2011. "Nutrition and Hydration Issues for Combat Sport Athletes." Strength and Conditioning Journal 33 (6): 10-7. 